\title{
Dose dependence of efficacy but not of safety in sublingual immunotherapy
}

\author{
F. Frati1, 3, C. Incorvaia2, F. Marcucci1, L. Sensi1, G. Di Cara1, \\ P. Puccinelli3, S. Dal Bo4
}

ABSTRACT: Dose dependence of efficacy but not of safety in sublingual immunotherapy. F. Frati, C. Incorvaia, F. Marcucci, L. Sensi, G. Di Cara, P. Puccinelli, S. Dal Bo.

Sublingual immunotherapy (SLIT) currently represents, as indicated by meta-analysis of its efficacy and safety, a valid option to the generally used traditional subcutaneous immunotherapy (SCIT) for treating respiratory allergy. Regarding efficacy, recent studies demonstrated that, similar to what has already been observed in SCIT as well as in experimental and clinical studies about the magnitudo of allergen exposure, the effectiveness on both clinical symptoms and immunologic changes depends on the amount of allergen administered during treatment.

In addition, in vitro studies addressed with the role of dendritic cells, currently considered to be of pivotal importance in orienting toward tolerance the immune response to allergens, showed that the internalisation of allergen molecules, which is followed by tolerogenic presentation to $T$ cells, depends on the amount of allergen.

However, such dose dependence is not apparent concerning the safety. In fact, the comparison of studies respectively conducted with high and low allergen doses did not show differences in the rate of systemic reactions, which in any case never had the presentation of anaphylaxis, and instead a significant difference in the rate of local reactions, following the oral and gastrointestinal contact with the allergen extract, in favour of high dose studies. Monaldi Arch Chest Dis 2006; 65: 1, 38-40.

Keywords: Sublingual immunotherapy, subcutaneous immunotherapy, asthma, rhinitis.

1 University Department of Obstetric, Gynaecologic and Pediatric Sciences, Perugia

2 Allergy/Pulmonary rehabilitation, Istituti Clinici di Perfezionamento, Milan

3 Scientific Department, Stallergenes Italia, Milan

4 Private allergy practice, Milan, Italy.

Correspondence: Franco Frati; Via G. Verga $n^{\circ}$ 3; Cortona; 52042 Arezzo Italy; e-mail: fratif@technet.it

\section{Introduction}

Sublingual immunotherapy (SLIT) was introduced as a further effort to improve the safety of hyposensitisation to inhalant allergens, which emerged in the eighties as a critical issue of the conventional subcutaneous route $[1,2]$. Previous attempts with other non injection routes did not meet such a need, because of inefficacy regarding the oral route and because of the impractical administration regarding the nasal route [3]. The sublingual route, by which the allergen extract is kept under the tongue for a few minutes and then swallowed, has instead accumulated increasing evidence on its efficacy and safety in the last 15 years and is currently a viable option for subcutaneous immunotherapy (SCIT). In fact, if in the Position paper on immunotherapy from the European Academy of Allergology and Clinical Immunology published in 1993 was reported that "more conclusive data is needed before sublingual immunotherapy can be recommended as a routine treatment of allergic diseases" [4], already the 1998 WHO document stated that "adequately controlled and well designed studies provided evidence that this form of treatment can constitute a valid option to parenteral immunotherapy" [5]. Indeed, the most important achievement was ob- tained with the Cochrane meta-analysis, which after reviewing more than 20 controlled studies concluded that "SLIT is a safe treatment which significantly reduces symptoms and medication requirements" [6].

Due to the importance of dose dependence in conventional SCIT, it is useful to consider this issue in SLIT under the two aspects of efficacy and safety.

\section{Dose dependence of efficacy of SLIT}

First studies on SLIT were conducted with low allergen doses but following the introduction of high dose allergen extracts it became apparent that the latter was more clinically effective. In the 2001 consensus document ARIA (Allergic Rhinitis and Its Impact on Asthma) the available data led to the statement "efficacy of high dose sublingual immunotherapy (at least 50-100 times higher than the cumulative dose of subcutaneous immunotherapy) was documented in double-blind placebo controlled controlled studies. Lower doses are not effective" [7].

Recent data strengthened such concept: a placebo-controlled study on patients suffering from ragweed-induced rhinoconjunctivitis reported that after five months of SLIT only the high 
dose - about 400 times higher than the standard dose administered with SCIT - was effective on nose and eye symptoms and on antihistamines consumption, while the low dose did not differ from placebo treatment [8].

With the same ragweed model, a trial conducted in Canada demonstrated that high dose SLIT is effective also with a co-seasonal schedule, since after only two weeks of treatment actively treated patients had symptom-medication scores significantly lower than placebo treated patients [9].

The relevance of direct comparison between SLIT and SCIT is obvious, and the study by the Malling group conducted in a double-blind double-dummy fashion (with patients respectively treated with placebo SLIT plus active SCIT, active SLIT plus placebo SCIT, and double placebo) found that SLIT with a birch extract with a dose 200 times higher than SCIT was as effective than SCIT on allergic symptoms during the birch pollination peak in February [10].

It is interesting to note that high dose SLIT seems to share with SCIT some outcomes of clinical efficacy: we observed, in children allergic to grass pollen treated with SLIT with a cumulative dose 375 times higher than the standard dose of SCIT, a blunting of the seasonal rise of pollen-specific IgE measured in nasal secretion [11], as generally observed in serum with effective SCIT [12]. At the same time these patients had lower symptom-medication scores, while children treated with a cumulative dose only 85 times higher than SCIT did not show such clinical and immunological outcome.

\section{Lack of dose dependence of safety of SLIT}

With SCIT, the need to administer high allergen doses to provide clinical efficacy is counterbalanced by the risk of adverse reactions, similarly dose-dependent [13]. It is reasonable to argue that also with other routes a dose dependence of safety would occur, and in fact it was reported with the oral and respiratory routes [3]. By contrast, there is no dose dependence of safety with SLIT, which has his typical adverse reaction in local disturbances, often transient and self-remitting, at oral and gastrointestinal contact with the allergen extract. In some studies conducted with low doses respiratory reactions were observed [14-16] but it is difficult to establish if such reactions were caused by the treatment or by the insufficient control of the disease.

An analysis published in 2000 [17] of the controlled studies available at that time which used high dose extracts - included one study administering a dose 500 times higher than SCIT [18] - reported a very good safety profile of active treatment compared to placebo and, of particular importance, no anaphylactic reactions.

In the aforementioned study by the Malling group, directly comparing SLIT to SCIT with a 200 times ratio, there were five systemic reactions and one anaphylactic reaction to SCIT in front of no systemic reaction at all with SLIT [10].
However, the most complete appraisal of SLIT safety was recently provided by an analysis which considered 25 controlled studies and compared low dose to high dose trials [19]. The findings showed that severe systemic reactions were absent and that there was no significant difference in the rate of slight/moderate systemic reactions between the two regimens. Instead, a significant difference was found in the rate of local reactions, which were 209 in 445 patients treated with high dose compared to 423 in 302 patients treated with low doses $(p<0.0001)$. Such a difference is difficult to explain, but at least two hypotheses can be posed, one suggesting that low amounts of allergen molecules may interact more efficiently with the specific $\operatorname{IgE}$ in the oral mucosa, as occurs in the socalled oral allergy syndrome [20], and the other considering that the reporting of local reactions may have a variable level of accuracy in the different studies, being possible to overlook them as negligible.

In addition, the safety in pediatric application is very satisfactory [21] and this led to verification of the possibility of applying SLIT in children aged less than 6 years, generally considered in consensus documents not eligible for allergen immunotherapy $[4,5]$. A recent study conducted with high dose SLIT in children aged 3 to 7 years found a similar rate of reactions (not severe enough to require discontinuation of treatment) in subjects aged 5 years or less compared to older subjects [22].

\section{Immunologic mechanisms underlying the dose-dependence}

Definite demonstrations of the mechanism of action of SLIT are not yet available, but increasing evidence attributes to dendritic cells a central role in the response to allergen molecules reaching the mouth. These cells are sparsely distributed in the mucosa including the external epithelial layer and show the anatomic features of the Langerhans cells present in various areas of the body with the function of antigen presenting cells. They are characterised by the expression of the high affinity $\operatorname{IgE}$ receptor FcepsilonRI related in atopic subjects to serum IgE levels [23]. The link of dendritic cell's IgE receptors, including the low affinity receptor CD23, with the allergen molecules elicits the production of a cytokine pattern which reduces the proliferation of Th2 cells, with a particular role for generation of regulatory $\mathrm{T}$ cells [24]. In an in vitro model it was observed that the internalisation of allergen molecules and control proteins were dose and time dependent and related to the immature state of the cells, with a higher efficiency for allergens, namely Bet v1 and Phl p 1 [24], which is likely to depend on the linking by the Fcepsilon RI receptor [25].

Thus, it seems reasonable to conceive that following the contact of the oral mucosa with the allergen administered with SLIT there is an activation of dendritic cells, which when reaching the most superficial layers phagocytate the allergen by 
macropinocytosis and transport it to the lymph nodes draining the anatomical area. Once migrated in the lymph nodes, dendritic cells express costimulatory molecules and interact with the naïve CD8 and CD4 T cells, a number of factors (including the allergen amount) being critical in determining a $\mathrm{T}$ response or tolerance.

Dr. Frati is Scientific Director of Stallergenes Italia.

\section{References}

1. Committee on Safety in Medicine. CSM update: desensitizing vaccines. BMJ 1986: 293: 948.

2. Lockey RF, Benedict LM, Turkeltaub PC, et al. Fatalities from immunotherapy and skin testing. J Allergy Clin Immunol 1987; 79: 660-77.

3. Canonica GW, Passalacqua G. Noninjection routes for immunotherapy. J Allergy Clin Immunol 2003; 111: 437-48.

4. Malling HJ, Weeke B (eds). Immunotherapy. Position Paper of the European Academy of Allergology and Clinical Immunology. Allergy 1993; 48 (suppl 14): 9-35.

5. WHO Position Paper. Allergen immunotherapy: therapeutic vaccines for allergic diseases. Allergy 1998; 53 (Suppl 54): 4-30.

6. Wilson DR, Torres-Lima M, Durham S. Sublingual immunotherapy for allergic rhinitis: systematic review and meta-analysis. Allergy 2005; 60: 4-12.

7. Bousquet J, van Cauwenberge P (eds). WHO initiative. Allergic rhinitis and its impact on asthma (ARIA). $J$ Allergy Clin Immunol 2001; 108 (suppl 5): 147-334.

8. André C, Perrin-Fayolle M, Grosclaude M, et al. A double-blind placebo-controlled evaluation of sublingual immunotherapy with a standardized ragweed extract in patients with seasonal rhinitis. Evidence for a dose-response relationship. Int Arch Allergy Immunol 2003; 131: 111-8.

9. Bowen T, Greenbaum J, Charbonneau Y, et al. Canadian trial of sublingual swallow immunotherapy for ragweed rhinoconjunctivitis. Ann Allergy Asthma Immunol 2004; 93: 425-430.

10. Khinchi MS, Poulsen LK, Carat F, André C, Hansen $\mathrm{AB}$, Malling HJ. Clinical efficacy of sublingual and subcutaneous birch pollen allergen-specific immunotherapy: a randomized, placebo-controlled, double blind, double dummy study. Allergy 2004; 59: 45-53.

11. Marcucci F, Sensi L, Di Cara G, Incorvaia C, Frati F. Dose dependence of immunological response to sublingual immunotherapy. Allergy 2005; 60: 952-6.

12. Van Metre TE, Adkinson NF Jr. Immunotherapy for aeroallergen disease. In Middleton E, Reed CE, Ellis EF, Adkinson NF Jr, Yunginger JW, Busse WW (eds):
Allergy. Principles and practice. St. Louis, Mosby-Year Book 1993: 1489-1509.

13. Turkeltaub PC. The importance of allergen dose on the safety and efficacy of immunotherapy of ragweed hay fever with standardized short ragweed extract. J Allergy Clin Immunol 1986; 77: 211-16.

14. Tari MG, Mancino M, Monti G. Efficacy of sublingual immunotherapy in patients with rhinitis and asthma due to house dust mite. a double-blind study. Allergol Immunopathol 1990; 18: 277-84.

15. Horack F, Stubner P, Berger UE, et al. Immunotherapy with sublingual birch pollen extract. a short-term double-blind placebo study. J Invest Allergol Clin Immunol 1998; 8: 165-71.

16. Voltolini S, Modena P, Minale P, et al. Sublingual Immunotherapy in tree pollen allergy. Double-Blind, Placebo-Controlled Study with a biologically standardised extract of three pollen (Alder, Birch, And Hazel) administered by a Rush Schedule. Allergol Immunopathol 2001; 29: 103-10.

17. Andé C, Vatrinet C, Galvain S, Carat F, Sicard H. Safety of sublingual-swallow immunotherapy in children and adults. Int Arch Allergy Immunol 2000; 121: 229-234.

18. Clavel R, Bousquet J, André C. Clinical Efficacy of sublingual-swallow immunotheraphy: a double-blind, placebo-controlled trial of a standardized five-grasspollen extract in rhinitis. Allergy 1998; 53: 493-8.

19. Gidaro G, Marcucci F, Sensi L, Incorvaia C, Frati F, Ciprandi G. The safety of sublingual-swallow immunotherapy: an analysis of published studies. Clin Exp Allergy 2005; 35: 565-71.

20. Pastorello EA, Incorvaia C, Ortolani C. The mouth and pharynx. In Ortolani $\mathrm{C}(\mathrm{Ed})$ : Atlas on mechanisms in adverse reactions to foods. Allergy 1995; 50 (Suppl 20): 41-44.

21. Di Rienzo V, Pagani A, Parmiani S, Passalacqua G, Canonica GW. Post-marketing surveillance study on the safety of sublingual immunotherapy in children. $A l$ lergy 1999; 54: 1110-13.

22. Fiocchi A, Pajno GB, La Grutta S, et al. Safety of sublingual immunotherapy in children aged less than 6 years. Ann Allergy Asthma Immunol 2005; 95: 254-58.

23. Allam JP, Novak N, Fuchs C, et al. Characterization of dendritic cells from human oral mucosa: a new Langerhans cell type with high constitutive Fcepsilon RI expression. J Allergy Clin Immunol 2003; 112: 141-48.

24. Groux H, Fournier N, Cottrez F. Role of dendritic cells in the generation of regulatory T cells. Semin Immunol 2004; 16: 99-106.

25. Noirey N, Rougier N, André C, Schmitt D, Vincent C. Langerhans-like dendritic cells generated from cord blood progenitors internalize pollen allergens by macropinocytosis, and part of the molecules are processed and can activate autologous naive T lymphocytes. J Allergy Clin Immunol 2000; 105: 1194-201. 